\title{
Infrared Cancellation and Measurement
}

\author{
Michael E. Miller ${ }^{\dagger}$
}

Quantum field theories containing massless particles such as photons and gluons are divergent not just in the ultraviolet, but also in the infrared. Infrared divergences are typically regarded as less conceptually problematic than ultraviolet divergences because there is a reasonably straightforward cancellation mechanism that renders measurable physical observables such as decay rates and cross-sections infrared finite. In this paper, I scrutinize the restriction to measurable physical observables that is required to make the cancellation mechanism applicable. I argue that this restriction does not necessitate a retreat to operationalism about the meaning of the theory as one might reasonably have worried, but it does call attention to a collection of underappreciated conceptual issues lurking in the infrared regime of quantum field theories with massless particles.

1. Introduction. The structural core of non-relativistic quantum mechanics is reasonably well agreed upon. It includes states defined on a Hilbert space, operators on that space to represent observables, the Schrödinger dynamics, and the Born rule for determining probabilities for the outcomes of experiments. ${ }^{1}$ This structural core provides an algorithm for extracting empirical predictions from the theory. Interpretive debates are concerned with whether we should adopt an operationalist view of this algorithm, or if the structural core should be furnished with a realistic interpretation. And of course, providing such a realistic interpretation requires that one provide a resolution to the quantum measurement problem.

Giving a realistic interpretation of quantum field theory similarly requires a solution to the quantum measurement problem, but the measurement problem is often conspicuously absent in foundational discussions of the theory. One reason for this is that relativistic constraints raise difficulties for generalizing some solutions to the measurement problem from quantum mechanics to quantum field theory. Another reason is that quantum field theory is often characterized as a theory of scattering. ${ }^{2}$ This can be seen from the fact that the basic phenomenological object in the theory is often taken to be the S-matrix which encodes transition amplitudes between prepared incoming states and measured outgoing states, both with determinate particle content.

\footnotetext{
Draft of 3 July 2020

${ }^{\dagger}$ Department of Philosophy, University of Toronto

${ }^{1}$ Helpful critical discussion of what belongs to the structural core, and what does not, can be found in (Wallace 2019).

${ }^{2}$ The historical reasons for this are discussed in (Blum 2017).
} 
So one might worry that before we even get to the issue of the measurement problem, the formalism for the theory is tinged with operationalism. The structure of the theory is designed to capture the scattering experiments used to test the theory from the outset.

Suppose we are interested in pressing on and attempting to give a realist interpretation of the scattering phenomena that quantum field theory is able to describe. We can use the scattering form of Born's rule,

$$
\operatorname{Pr}\left(\psi_{\text {out }} \mid \psi_{\text {in }}\right):=\left|\left\langle\psi_{\text {out }}|S| \psi_{\text {in }}\right\rangle\right|^{2},
$$

to determine the probability of a transition from the state $\left|\psi_{\text {in }}\right\rangle$ to the state $\left|\psi_{\text {out }}\right\rangle$. On first inspection, this seems to involve essentially the same structural core as non-relativistic quantum mechanics, and to provide an algorithm for predicting the outcomes of experiments which we can go about interpreting. However, the quantum field theoretic algorithm is beset with interpretive challenges of its own that arise before we confront the measurement problem. As a result, much of the interpretive work dedicated to quantum field theory has been concerned with the processes that are required to get the algorithm up and running, and not the interpretation of the algorithm itself.

The interpretive difficulties facing the quantum field theoretic algorithm are diverse. For one, $\left|\psi_{\text {in }}\right\rangle$ and $\left|\psi_{\text {out }}\right\rangle$ are not states in the physical statespace of the interacting quantum fields involved in the scattering. Rather, they are states in the statespace of free fields. Information about the interacting fields must be gleaned from the perturbative evaluation of the S-matrix element for a particular $\left|\psi_{\text {in }}\right\rangle$ and $\left|\psi_{\text {out }}\right\rangle$. To do this we sum all of the Feynman diagrams with the appropriate particle content and incoming and outgoing momenta. This perturbative evaluation gives rise to additional obstacles to interpretation. The most widely discussed of these are the ultraviolet divergences that arise from the short-distance and large-momentum regime of the theory. The integrals corresponding to individual diagrams contributing to the probabilities in Eq. (1) are infinite. These ultraviolet divergences necessitate the renormalization of the theory in order to render predictions for the outcomes of experiments finite. ${ }^{3}$ Some presentations of the theory give the impression that a properly implemented renormalization procedure is sufficient to get an algorithm up and running that gives probabilities that match the experimental results.

\footnotetext{
${ }^{3}$ With the development of the renormalization group, the physical need for this process is now well-understood. Quantum field theories are understood as effective theories with an explicitly specified domain of applicability. Recent philosophical literature has begun to address how this approach to understanding the ultraviolet divergences might affect the prospects for realist interpretations of the the theory. For my purposes, the important conclusion that can be drawn from these discussions is that the ultraviolet divergences do not provide an obstacle to realist interpretations of field theory.
} 
In fact, an additional step is required. There is an independent source of infinities that need to be addressed before the algorithm yields finite probabilities. These infrared divergences come from the long-distance and smallmomentum regime of the theory, and have received comparatively little attention in the literature. The infrared divergences result from the emission of very low momentum massless particles, and are typically regarded as less conceptually problematic than ultraviolet divergences because there is a reasonably straightforward cancellation mechanism that renders physical observables such as decay rates and cross-sections infrared finite. More precisely, the infrared divergences cancel when we restrict to measurable physical quantities. My aim in this paper is to scrutinize the restriction to measurable physical observables that is required to make the cancellation mechanism applicable. It is prima facie plausible that there are physical quantities that are not measurable, but about which there are still facts. For this reason, a restriction to what is measurable is potentially problematic. If one adopts an operationalist interpretation which only countenances those quantities which are measurable as meaningful, such a restriction is unproblematic. However, if one ultimately aspires to provide a realist interpretation, one needs the quantum field theoretic algorithm to be well-defined for all of the physically meaningful quantities, which may not just be the measurable ones. So to ensure that the restriction in question does not amount to a thumb on the operationalist's side of the scale, we need to make sure that we are not restricting beyond the physical matters of fact.

In order to determine whether or not the restriction to measurable physical quantities is an acceptable one, we must analyze the origin of the infrared divergences and the infrared cancellation mechanism in detail. I turn to that task in Section Two. In Section Three I discuss the restriction to measurable physical quantities and I argue that it need not mark a problematic retreat to operationalism. In the fourth section I argue that the infrared divergences from massless particles are a conceptually distinct infrared problem from the one raised by Haag's theorem. The infrared divergences discussed here are more directly relevant for the prospects of providing a realist interpretation of the theory because they bear on the nature of the physical statespace of the theory. Section Five concludes by emphasizing that the infrared regime of quantum field theory contains foundationally significant issues which are important for the project of interpreting the theory.

2. Infrared Cancellation. Early in the development of quantum electrodynamics it was recognized that the infrared problems of classical electrodynamics carried over to quantum field theory. In this latter context, the problems stem from the presence of massless particles. If a massless particle is "soft" in the sense that it has very low momentum, then the emission of such a particle requires very little energy. In the case of quantum electrody- 
namics, for example, in processes with outgoing electrons in the final state, the electron is never actually free as we are accustomed to thinking of it. In reality, outgoing electrons emit many soft photons which lead to infrared divergences in the S-matrix element for the process. ${ }^{4}$ Closely analogous problems arise in quantum chromodynamics due to the massless gluons, and in quantum theories of gravity involving massless gravitons.

An approach to addressing the infrared divergences was discovered by Bloch and Nordsieck even before the development of covariant perturbation theory for quantum electrodynamics (Bloch and Nordsieck 1937). What they realized was that the infrared divergences from the emission of soft photons are perfectly cancelled by infrared divergences from virtual soft photons. This cancellation mechanism was elaborated in full detail for quantum electrodynamics by Yennie, Frautschi and Suura who showed conclusively that QED can be rendered infrared finite to all orders of perturbation theory (Yennie, Frautschi, and Suura 1961). Weinberg produced a significant simplification of the argument, which also applies to theories with massless gravitons, shortly after (Weinberg 1965). Similar arguments, though more limited in their generality, have also been provided for quantum chromodynamics. ${ }^{5}$ The central observation required to induce the cancellation in each case is that any realistic particle detector has some minimum energy threshold. Particles with energy below this threshold will pass through the detector undetected. When S-matrix elements, transition rates, and cross-sections are expressed in a way that accounts for the presence of such a threshold, the infrared divergences can be shown to cancel to all orders.

Suppose we are interested in a QED process with initial state $\alpha$ and final state $\beta$ containing a total of $n$ incoming and outgoing electrons. ${ }^{6}$ The Smatrix element for this process $S_{\beta \alpha}$ requires corrections from the emission of soft photons. Consider the simplest case where a single soft photon is emitted from one of the outgoing electron lines as shown in Fig. 1(a). This yields a correction given by the product of an electron-photon vertex, and an electron propagator with momentum $p+q$, in the limit where $q \rightarrow 0:^{7}$

$$
\left[i(2 \pi)^{4} e\left(2 p^{\mu}+q^{\mu}\right)\right] \cdot\left[\frac{-i}{(2 \pi)^{4}} \frac{1}{(p+q)^{2}+m^{2}-i \epsilon}\right] \stackrel{q \rightarrow 0}{\longrightarrow} \frac{e p^{\mu}}{p \cdot q-i \epsilon} .
$$

\footnotetext{
${ }^{4}$ Additional infrared divergences can occur when massless particles move collinearly with the particle from which they were emitted. This class of divergences can be addressed with methods similar to those discussed in this section, though they will not be my focus in this paper.

${ }^{5}$ One important example is provided by the KLN theorem (Kinoshita 1962; Lee and Nauenberg 1964). For helpful discussion see (Muta 1987, Ch. 6).

${ }^{6}$ The argument I present here is a simplified version of the one initially given in (Weinberg 1965) and further elaborated in (Weinberg 1995, Ch. 13).

${ }^{7}$ In taking the limit I have used the freedom to rescale $\epsilon$ without changing the sign of the term.
} 


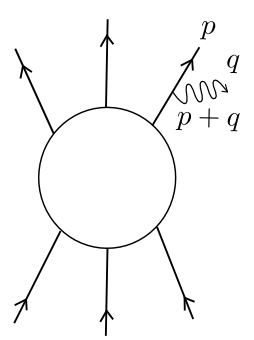

(a)

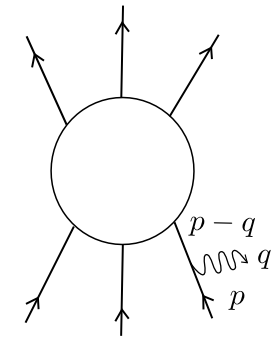

(b)

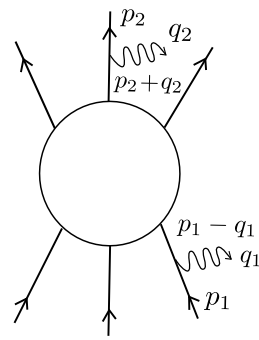

(c)

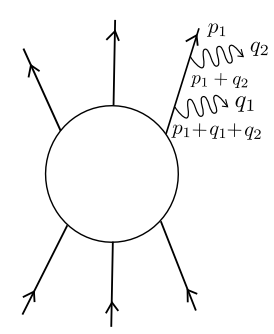

(d)

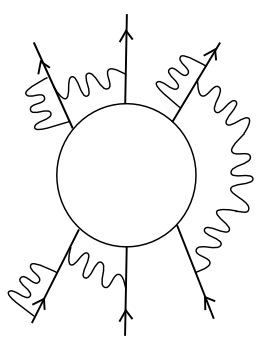

(e)

Figure 1: Emission of real soft photons, and exchange of virtual soft photons.

If the photon is emitted from an incoming line rather than an outgoing line, as shown in Fig. 1(b), then the momentum in the additional propagator is $p-q$ and the correction is given by:

$$
\left[i(2 \pi)^{4} e\left(2 p^{\mu}-q^{\mu}\right)\right] \cdot\left[\frac{-i}{(2 \pi)^{4}} \frac{1}{(p-q)^{2}+m^{2}-i \epsilon}\right] \stackrel{q \rightarrow 0}{\longrightarrow} \frac{e p^{\mu}}{-p \cdot q-i \epsilon} .
$$

To obtain the correction for the emission of a single soft photon from any of the incoming or outgoing electron lines we must sum over each way the process can happen. If we adopt the convention that $\eta_{n}=+1$ if the emission is from an outgoing line and $\eta_{n}=-1$ if it is from an incoming line, this sum can be written compactly as:

$$
\sum_{n} \frac{\eta_{n} e p_{n}^{\mu}}{p_{n} \cdot q-i \eta_{n} \epsilon}
$$

If two soft photons are emitted, the correction is given by a product of factors like those we found in Eq. (2) and Eq. (3). For example, if one is emitted from an incoming line and one is emitted from an outgoing line, as in Fig. 1(c), the correction is given by:

$$
\left[\frac{e p_{2}^{\mu}}{p_{2} \cdot q_{2}-i \epsilon}\right] \cdot\left[\frac{e p_{1}^{\mu}}{-p_{1} \cdot q_{1}-i \epsilon}\right] .
$$

If both electrons are emitted from the same outgoing line, as in Fig. 1(d), then the correction is:

$$
\left[\frac{e p_{1}^{\mu}}{p_{1} \cdot q_{2}-i \epsilon}\right] \cdot\left[\frac{e p_{1}^{\mu}}{p_{1} \cdot\left(q_{1}+q_{2}\right)-i \epsilon}\right]
$$

A simple induction ${ }^{8}$ shows that the correction for the emission of $N$ soft

\footnotetext{
${ }^{8}$ See (Weinberg 1995, pp. 538-539).
} 
photons is given by:

$$
\prod_{i=1}^{N}\left(\sum_{n} \frac{e \eta_{n} p_{n}^{\mu_{i}}}{p_{n} \cdot q_{i}-i \eta_{n} \epsilon}\right)
$$

From this basic relation we can determine the effects of both virtual and real soft photons on $S_{\beta \alpha}$.

To determine the correction from the contribution of soft virtual photons depicted in Fig. 1(e), we must introduce a scale $\Lambda$ which determines which virtual photons we want to count as soft. Different choices of $\Lambda$ simply correspond to different choices of what count as radiative corrections, and what count as part of the uncorrected matrix element. We will also be manipulating infrared divergent expressions and so we will introduce an infrared cutoff $\lambda$. This cutoff will eventually be removed by taking the $\lambda \rightarrow 0$ limit at the end of the calculation.

The correction from a single soft virtual photon can be determined by taking the product of two emitted photon corrections, multiplied by a photon propagator $\left(-i g_{\mu \nu}\right) /\left[(2 \pi)^{4} \cdot\left(q^{2}-i \epsilon\right)\right]$, summing over the polarization indices, and integrating over the soft photon momentum:

$$
\int_{\lambda}^{\Lambda} d^{4} q A(q)
$$

where,

$$
A(q)=\frac{-i}{(2 \pi)^{4}\left(q^{2}-i \epsilon\right)} \cdot \sum_{n, m} \frac{e^{2} \eta_{n} \eta_{m}\left(p_{n} \cdot p_{m}\right)}{\left.\left(p_{m} n \cdot q-i \eta_{n} \epsilon\right)\left(-p_{m} \cdot q-i \eta_{m} \epsilon\right)\right]}
$$

To obtain the correction from $N$ virtual soft photons, we take the product of $N$ such factors, and divide by factors of $N$ ! to account for possible permutations of where the lines attach, and $\left(2^{N}\right)$ to account for interchanges of the two ends of the line. This gives,

$$
\frac{1}{N !}\left[\frac{1}{2} \int_{\lambda}^{\Lambda} d^{4} q A(q)\right]^{N}
$$

and thus, when we sum over $N$ and use the fact that $\exp (x)=\sum_{N} x^{N} / N$ ! we find that,

$$
S_{\beta \alpha}^{\lambda}=S_{\beta \alpha}^{\Lambda} \exp \left(\frac{1}{2} \int_{\lambda}^{\Lambda} d^{4} q A(q)\right) .
$$

$S_{\beta \alpha}^{\Lambda}$ is the S-matrix element with no virtual photon exchange with momentum less than $\Lambda$ included. $S_{\beta \alpha}^{\lambda}$ is the S-matrix element corrected to include virtual soft photon exchange with momentum greater than $\lambda$ but less than $\Lambda$. The 
rate for the process is then given by the matrix element squared:

$$
\Gamma_{\beta \alpha}^{\lambda}=\left|S_{\beta \alpha}^{\lambda}\right|^{2}=\left|S_{\beta \alpha}^{\Lambda}\right|^{2} \exp \left(\int_{\lambda}^{\Lambda} d^{4} q A(q)\right)=\Gamma_{\beta \alpha}^{\Lambda} \exp \left(\int_{\lambda}^{\Lambda} d^{4} q A(q)\right) .
$$

Weinberg shows that the integral in the exponential yields:

$$
\int_{\lambda}^{\Lambda} d^{4} q A(q)=-A \ln \left(\frac{\Lambda}{\lambda}\right)
$$

where,

$$
A=\frac{-1}{8 \pi} \sum_{n, m} \frac{e^{2} \eta_{n} \eta_{m}}{\beta_{n m}} \ln \left(\frac{1+\beta_{n m}}{1-\beta_{n m}}\right) \text { and } \beta_{n m}=\left[1-\frac{m_{e}^{4}}{\left(p_{n} \cdot p_{m}\right)^{2}}\right]^{1 / 2} .
$$

Inserting Eq. (13) into Eq. (12), and using familiar properties of exponentials and logarithms, we find that:

$$
\Gamma_{\beta \alpha}^{\lambda}=\Gamma_{\beta \alpha}^{\Lambda} \exp \left(-A \ln \left(\frac{\Lambda}{\lambda}\right)\right)=\Gamma_{\beta \alpha}^{\Lambda}\left[\exp \left(\ln \left(\frac{\lambda}{\Lambda}\right)\right)\right]^{A}=\Gamma_{\beta \alpha}^{\Lambda}\left(\frac{\lambda}{\Lambda}\right)^{A} .
$$

This provides a complete statement of the correction to the rate from virtual soft photons. In the limit where $\lambda \rightarrow 0$ we see that the rate $\Gamma_{\beta \alpha}^{\lambda}$ vanishes. This is the result of exponentiating $\ln (\Lambda / \lambda)$ which is divergent in the $\lambda \rightarrow 0$ limit.

The virtual soft photon divergences leading to this unphysical vanishing of the rate are cancelled by divergences from real photon emission. More precisely, this cancellation can be seen to apply to all orders of perturbation theory when the total rate, including all radiative corrections, is expressed in terms of the resolution of the detector used to measure the real soft photons. Weinberg explains the restriction as follows:

The resolution of the infrared divergence problem .... is found in the observation that it is not really possible to measure the rate $\Gamma_{\beta \alpha}$ for a reaction $\alpha \rightarrow \beta$ involving definite numbers of photons and charged particles, because photons of very low energy can always escape undetected. What can be measured is the rate $\Gamma_{\beta \alpha}\left(E, E_{T}\right)$ for such a reaction to take place with no unobserved photon having an energy greater than some small quantity $E$, and with not more than some small total energy $E_{T}$ going into any number of unobserved photons. (Weinberg 1995, pp. 544-545, my emphasis)

This restriction to the measurable quantity $\Gamma_{\beta \alpha}\left(E, E_{T}\right)$ in order to render the rate infrared finite requires careful analysis. I will turn to that task in Section 
Three. The remainder of this section completes the demonstration that if one makes this restriction, then the infrared divergences cancel.

In order to calculate the correction from the emission of $N$ real soft photons, with momenta $q_{1}, \ldots, q_{N}$, each term in Eq. (7) must be multiplied by the appropriate coefficient function, ${ }^{9}$

$$
\frac{\epsilon_{\mu}^{*}\left(\mathbf{q}_{\mathbf{i}}, h_{i}\right)}{(2 \pi)^{3 / 2}\left(2\left|\mathbf{q}_{\mathbf{i}}\right|\right)^{1 / 2}} .
$$

This yields the following expression for the matrix element $S_{\beta \alpha}^{\lambda}\left(q_{1}, q_{2}, \ldots, q_{N}\right)$, which includes the contributions of both the virtual soft photons and the $N$ real emitted soft photons:

$$
S_{\beta \alpha}^{\lambda}\left(q_{1}, q_{2}, \ldots, q_{N}\right)=S_{\beta \alpha}^{\lambda} \prod_{i=1}^{N} \frac{1}{(2 \pi)^{3 / 2}\left(2\left|\mathbf{q}_{i}\right|\right)^{1 / 2}} \cdot \sum_{n} \frac{\eta_{n} e\left(p_{n} \cdot \epsilon^{*}\left(\mathbf{q}_{i}, h_{i}\right)\right)}{\left(p_{n} \cdot q_{i}\right)}
$$

where $S_{\beta \alpha}^{\lambda}$ is as given in Eq. (11). The differential rate for the emission of $N$ soft photons into the volume of momentum space $\prod_{i} d^{3} q_{i}$, is given by squaring Eq. (17), summing over the helicities, and multiplying by $\prod_{i} d^{3} q_{i}$ which gives:

$$
d \Gamma_{\beta \alpha}^{\lambda}\left(q_{1}, q_{2}, \ldots, q_{N}\right)=\Gamma_{\beta \alpha}^{\lambda} \prod_{i=1}^{N} \frac{d^{3} q_{i}}{(2 \pi)^{3}\left(2\left|\mathbf{q}_{i}\right|\right)} \cdot \sum_{n m} \frac{\eta_{n} \eta_{m} e^{2}\left(p_{n} \cdot p_{m}\right)}{\left(p_{n} \cdot q_{i}\right)\left(p_{m} \cdot q_{i}\right)}
$$

Integrating over the direction of photon propagation yields the differential rate for the emission of $N$ soft photons with energies $\omega_{1}, \ldots, \omega_{N}$ :

$$
d \Gamma_{\beta \alpha}^{\lambda}\left(\omega_{1}, \omega_{2}, \ldots, \omega_{N}\right)=\Gamma_{\beta \alpha}^{\lambda} A^{N} \frac{d \omega_{1}}{\omega_{1}} \frac{d \omega_{2}}{\omega_{2}} \cdots \frac{d \omega_{N}}{\omega_{N}}
$$

where the factor $A$ is as defined in Eq. (14). Note that if we were to integrate Eq. (19) over the emitted energies of the photons, we would produce logarithmic divergences from the $\omega \rightarrow 0$ end of the integrations. However, the imposition of the infrared cutoff $\lambda$ ensures that the expressions are regulated. If we were to remove the regulator at this stage of the calculation, the cancellation mechanism would not do its job, and we would not arrive at a sensible physical rate at the end of the calculation.

In order to arrive at a final expression for the rate, the integration over photon energies must be done respecting the constraints described in the quotation of Weinberg above. In particular, the unobserved photons must each have energy below the detector threshold and above the infrared cutoff, $E \geq \omega_{i} \geq \lambda$, and the total energy of all of the unobserved photons must not

${ }^{9}$ In this expression, $\epsilon$ is a polarization vector and $h$ is the helicity. 
be greater than $E_{T}, \sum_{i} \omega_{i} \leq E_{T}$ :

$$
\Gamma_{\beta \alpha}^{\lambda}\left(E, E_{T}\right)=\Gamma_{\beta \alpha}^{\lambda} \sum_{N=0}^{\infty} \frac{A^{N}}{N !} \int_{E \geq \omega_{i} \geq \lambda, \sum_{i} \omega_{i} \leq E_{T}} \prod_{i=1}^{N} \frac{d \omega_{i}}{\omega_{i}}
$$

The integration subject to these restrictions gives: ${ }^{10}$

$$
\Gamma_{\beta \alpha}^{\lambda}\left(E, E_{T}\right)=\left(\frac{E}{\lambda}\right)^{A} \Gamma_{\beta \alpha}^{\lambda}
$$

The cancellation of the infrared divergences is achieved by inserting the expression in Eq. (15) for $\Gamma_{\beta \alpha}^{\lambda}$ into Eq. (21). This combines all corrections from real and virtual photons into an expression for $\Gamma_{\beta \alpha}^{\lambda}\left(E, E_{T}\right)$ :

$$
\Gamma_{\beta \alpha}^{\lambda}\left(E, E_{T}\right)=\left(\frac{E}{\lambda}\right)^{A} \Gamma_{\beta \alpha}^{\lambda}=\left(\frac{E}{\lambda}\right)^{A}\left(\frac{\lambda}{\Lambda}\right)^{A} \Gamma_{\beta \alpha}^{\Lambda}=\left(\frac{E}{\Lambda}\right)^{A} \Gamma_{\beta \alpha}^{\Lambda} .
$$

Note that the factors of $\lambda$ cancelled each other, and so we can take $\lambda \rightarrow 0$ to obtain:

$$
\Gamma_{\beta \alpha}\left(E, E_{T}\right)=\left(\frac{E}{\Lambda}\right)^{A} \Gamma_{\beta \alpha}^{\Lambda}
$$

Thus, when we account for both soft virtual photon exchange and real soft photon emission, the rate becomes independent of $\lambda$ and is infrared finite. The procedure used to achieve this result does, however, introduce a dependence on the detector resolution, $E$.

The subsequent literature adopts a distinction between exclusive and inclusive quantities. ${ }^{11}$ Exclusive quantities stipulate the exact contents of the incoming and outgoing states. For example, in an exclusive cross-section one might demand that there are exactly three electrons and no other particles, even if the other particles are not detected. Inclusive quantities stipulate part of the contents of the final state, but they also account for the possibility that there are other particles in the final state. The rate in Eq. (23) provides an example of an inclusive quantity. We have stipulated that there are a total of $n$ incoming and outgoing electron lines, but we have also accounted for the emission of an arbitrary number of undetected soft photons each with energy less than $E$ and with total energy less than $E_{T}$. At particle accelerators, attention is often restricted to such inclusive quantities, and the it is the justification for this to which we now turn our attention.

\footnotetext{
${ }^{10} \mathrm{I}$ have omitted an overall factor resulting from the integration which is close to 1 in the circumstances we are interested in analyzing.

${ }^{11}$ As far as I have been able to determine, this distinction originates from (Feynman 1969).
} 
3. Measurement. The apparent need to restrict to measurable physical quantities has arisen in other contexts during the development of quantum field theory. Early in the development of the theory, Bohr and Rosenfeld argued that the value of the field at a point was not a measurable quantity, but that the average value of the field over a small spacetime region was measurable (Bohr and Rosenfeld 1933; Bohr and Rosenfeld 1950). It was later realized that field operators could not be mathematically defined at points of spacetime, and that instead they had to be represented as operatorvalued distributions which are well-defined only as integrations against test functions of compact support on small regions of spacetime. ${ }^{12}$ When it was realized that the mathematical definition of the theory became ill-defined for associations of operators with points, a number of figures suggested that this should be intepreted as resulting from the fact that such quantities were unmeasurable. ${ }^{13}$ If one adopts the additional assumption that unmeasurable quantities are not meaningful, then the ill-definedness of field operators at points becomes unproblematic: there is no physically meaningful quantity for the ill-defined field operators to correspond to.

Similar reasoning has been employed to address other ill-defined quantities from the ultraviolet regime. Empirically interesting field theories are ultraviolet divergent and require renormalization. This process involves recognizing that some parameters in the lagrangian such as the bare mass and the bare charge are infinite and introducing counterterms to cancel the infinities and re-express the theory in terms of measurable parameters such as the dressed mass and charge. In response to this situation one frequently encounters the claim that bare parameters in the Lagrangian are unmeasurable. To take just one example, Srednicki explains that "It may be disturbing to have a parameter in the Lagrangian that is formally infinite. However, such parameters are not directly measurable, and so need not obey our preconceptions about their magnitudes" (Srednicki 2007, p. 67). ${ }^{14}$ Once again, we encounter the view that only those quantities that are measurable are required to be meaningful.

Compare this to the reasoning Weinberg offered in the previous section. The rate $\Gamma_{\beta \alpha}^{\lambda}$ is infrared divergent in the limit where $\lambda \rightarrow 0$, but it is unmeasurable. The measurable rate $\Gamma_{\beta \alpha}^{\lambda}\left(E, E_{T}\right)$ is infrared finite to all orders of perturbation theory in the $\lambda \rightarrow 0$ limit. The justification for the need to make this restriction in order to arrive at infrared finite quantities, when one is explicitly articulated, is that any real physical detector has some finite energy resolution and particles with energy below that threshold will not be

${ }^{12}$ This came to be understood in stages, with the conclusive theorem provided in (Wightman 1964).

${ }^{13}$ (Friedrichs 1951; Cook 1953)

${ }^{14}$ Similar claims can be found in (Peskin and Schroeder 1995, p. 315) and (Itzykson and Zuber 2012, p. 319), and in many other accounts of the rationale underlying renormalization. 
registered in the detector. Thus, Weinberg's demonstration establishes that quantum field theory provides well-defined values for all of those observables that are physically measurable and most discussions of this issue leave off here. ${ }^{15}$

Absent additional argumentation, I think that this amounts to a problematic retreat to operationalism. To be clear, my concern is not with operationalism as an account of meaning in general. I am open to the possibility that operationalism provides a compelling account of meaning in at least some cases. What is problematic in this case is that the justification for the restriction to measurable quantities relies on the stronger claim that only those quantities that are measurable are physically meaningful. Suppose this stronger claim were true. Then the demonstration that the field theoretic expressions for the measurable observables are well-defined amounts to a demonstration that the field theoretic expressions for every physically meaningful quantity is well-defined. If the stronger claim is not true, and there are physically meaningful quantities that are not measurable, then the demonstration that the measurable quantities are well-defined does not go far enough to establish that the theory adequately accounts for all of the meaningful quantities.

To determine whether or not the restriction to measurable quantities in the infrared case is problematic, we need to know whether or not failures of measurability stand in direct correspondence with failures of meaningfulness. For this reason, each proposed restriction to measurable quantities requires its own analysis, as each involves distinct physical limitations on what is measurable. While I believe that both of the ultraviolet cases introduced above merit further attention of their own, here I will restrict attention to the infrared case as that is my central concern in this paper.

Suppose we simply grant that every physical detector will have some threshold $E$ such that particles with energy less than $E$ will not be detected. ${ }^{16}$ Note that quantities like cross-sections and rates are defined with respect to a particular collection of incoming particles, and a particular collection of detected outgoing particles. However, for a given incoming state, $\alpha$, the dynamics of the theory will yield an outgoing state which is a superposition with indeterminate particle content, including an indeterminate number of electrons, hard photons, and soft photons with energy below the detection threshold. It is only upon measurement that the outgoing state becomes

\footnotetext{
${ }^{15}$ Essentially the same justification can be found throughout the physics literature. See, for example, (Brown 1992, pp. 490-491), (Duncan 2012, p. 719, p. 723, p. 728), (Itzykson and Zuber 2012, p. 173, p. 354), (Peskin and Schroeder 1995, pp. 200-202), (Schweber 2011, p. 549), and (Srednicki 2007, pp. 157-158).

${ }^{16}$ This claim is often asserted without argument. Establishing its validity would require a detailed analysis of the physical nature of the detector and its coupling to the measured particle. I am grateful to Jeff Barrett for discussion of this point.
} 
one with the determinate particle content as we assumed $\beta$ to have. And, of course, how one conceives of this process of becoming a state with determinate particle content depends on how one prefers to resolve the quantum measurement problem.

In computing the rate $\Gamma_{\beta \alpha}\left(E, E_{T}\right)$ we assumed that this measurement process yields a specific number of electrons and no hard photons in the final state. If there were hard photons, or a different number of electrons, we would need to compute the rate for a different process. Given that there are outgoing electrons in the final state, there are also soft photons which were not detected. So the justification relied on here is not that there is no photon detector that can detect arbitrarily soft photons and hence quantities involving them are meaningless. Rather, every measurement that is done has some energy resolution, and we need to account for the fact that given the particular measurement that has been executed, there can be soft photons below that resolution.

This shows why it necessary to express the physical quantities in terms of the detector resolution, $E$. For a given incoming state, there are distinct possible outgoing states. By selecting a specific $\beta$, we have not done quite enough to specify which part of the statespace the measurement is a projection onto. By specifying $E$, we condition on which kinds of soft radiation can be undetected in the final state. For a different detector energy resolution $E^{\prime}$, different kinds of unobserved soft radiation states are possible, as are different alternatives to $\beta$. The need to restrict to what is measurable is not a retreat to operationalism. Rather, the presence of the energy resolution is an articulation of the precise nature of the question we are asking about the outgoing state by executing the particular measuring process that we chose to execute.

4. The Connection to Haag's Theorem. In their appraisal of the philosophical significance of Haag's theorem, Earman and Fraser make several references to infrared divergences (Earman and Fraser 2006). They claim, for example, that "In the physicists' lingo, the move from one inequivalent representation to another is marked by divergences. Haag's theorem is concerned with infrared divergences that are associated with Euclidean invariance and the infinite volume of space (Earman and Fraser 2006, p. 319)". They also note the infrared divergences can be tamed by imposing some form of infrared regulator. ${ }^{17}$ The imposition of an infrared regulator can cure more than one kind of infrared pathology, and caution is required here in order not to run together two conceptually distinct issues.

The interaction picture is a formal intermediary between the the Schrödinger picture and the Heisenberg picture which is often employed as a calculational

${ }^{17}$ The regulators they consider are the compactification of space, and the restriction of the theory to bounded regions of spacetime (Earman and Fraser 2006, p. 319, 323, 330). 
tool to facilitate the perturbative evaluation of observables. It postulates the existence of a global unitary transformation connecting the free and interacting Hilbert spaces. Haag's theorem shows that this transformation does not exist and that these spaces are unitarily inequivalent. Thus, the interaction picture is predicated on an inconsistent set of assumptions. Miller has provided an account of how perturbative calculations that employ the interaction picture can be empirically successful despite this apparent inconsistency (Miller 2016). The imposition of an infrared regulator renders some of the assumptions of the theorem false. This undercuts the threat to the empirical success of the theory from Haag's theorem, but it leaves questions about the well-definedness of the interaction picture in the limit where the regulator is removed.

Infrared divergences from soft massless particles raise a more serious worry about the infrared regime of quantum field theory than the one implicated in Haag's theorem. The infrared cancellation results are sufficient to assuage worries about how it can be that theories with infrared divergences are still empirically successful. However, because of the presence of the soft massless particles, free electron states with distinct momenta are unitarily inequivalent to one another. ${ }^{18}$ As such, this class of infrared divergences call into question the well-definedness of the physical state spaces of theories like quantum electrodynamics. For this reason, I think they are rightly regarded as a symptom of more serious conceptual problem than Haag's theorem, which only undermines a method for extracting predictions from the theory. The challenge from the soft massless particles is a serious one for interpreters of quantum field theory and it is one which in my view requires significant further attention. ${ }^{19}$

5. Conclusion. I have argued that the need to express physical quantities in terms of the energy resolution of a detector does not mark a problematic retreat to operationalism. As in the case of the ultraviolet divergences, the infrared divergences can be understood physically. With a properly implemented renormalization scheme and infrared cancellation mechanism in place, the algorithm of quantum field theory provides finite expressions for physical observables. Thus, the infrared divergences, like the ultraviolet divergences, are not ultimately an obstacle to realist interpretations of the theory. The infrared regime of the theory is fraught with conceptual issues which bear directly on the issue of how one might go about producing such an interpretation, and very much warrants further attention from a foundational perspective.

\footnotetext{
${ }^{18}$ For discussion see (Duncan 2012, pp. 722-723) or (Buchholz 1982).

${ }^{19}$ Perhaps the first philosopher to approach this problem is Ruetsche, who has suggested that coherent state representations may play an important role in understanding these issues (Ruetsche 2012, pp. 245-246).
} 


\section{References}

Bloch, F. and A. Nordsieck (1937). Note on the Radiation Field of the Electron. Phys. Rev. 52, 54-59.

Blum, A. S. (2017). The state is not abolished, it withers away: How quantum field theory became a theory of scattering. Stud. Hist. Phil. Sci. B60, 46-80.

Bohr, N. and L. Rosenfeld (1933). Zur frage der messbarkeit der elektrimagnetischen feldgrössen. Det Kgl. Danske Videnskabernes Selskab. Mathematisk-fysiske Meddelelser 12, 1-65.

Bohr, N. and L. Rosenfeld (1950). Field and charge measurements in quantum electrodynamics. Phys. Rev. 78, 794-798.

Brown, L. S. (1992). Quantum Field Theory. Cambridge University Press.

Buchholz, D. (1982). The physical state space of quantum electrodynamics. Comm. Math. Phys. 85(1), 49-71.

Cook, J. M. (1953). The mathematics of second quantization. Transactions of the American Mathematical Society $74(2), 222-245$.

Duncan, A. (2012). The conceptual framework of quantum field theory. Oxford: Oxford Univ. Press.

Earman, J. and D. Fraser (2006). Haag's Theorem and its Implications for the Foundations of Quantum Field Theory. Erkenntnis 64.

Feynman, R. P. (1969). Very high-energy collisions of hadrons. Phys. Rev. Lett. 23, $1415-1417$.

Friedrichs, K. O. (1951). Mathematical aspects of the quantum theory of fields parts i and ii. Communications on Pure and Applied Mathematics 4(2-3), 161-224.

Itzykson, C. and J. Zuber (2012). Quantum Field Theory. Dover Books on Physics. Dover Publications.

Kinoshita, T. (1962). Mass singularities of Feynman amplitudes. J. Math. Phys. 3, 650677.

Lee, T. D. and M. Nauenberg (1964). Degenerate Systems and Mass Singularities. Phys. Rev. 133, B1549-B1562.

Miller, M. E. (2016). Haag's Theorem, Apparent Inconsistency, and the Empirical Adequacy of Quantum Field Theory. The British Journal for the Philosophy of Science $69(3), 801-820$.

Muta, T. (1987). Foundations of quantum chromodynamics: An Introduction to perturbative methods in gauge theories. World Sci. Lect. Notes Phys. 5, 1-409.

Peskin, M. and D. Schroeder (1995). An Introduction To Quantum Field Theory. Frontiers in Physics. Avalon Publishing.

Ruetsche, L. (2012). Interpreting Quantum Theories. Oxford: Oxford Univ. Press.

Schweber, S. (2011). An Introduction to Relativistic Quantum Field Theory. Dover Publications.

Srednicki, M. (2007). Quantum Field Theory. Cambridge University Press.

Wallace, D. (2019). What is orthodox quantum mechanics? In A. Cordero (Ed.), Philosophers Look at Quantum Mechanics. Springer Verlag.

Weinberg, S. (1965). Infrared photons and gravitons. Phys. Rev. 140, B516-B524.

Weinberg, S. (1995). The Quantum Theory of Fields, Volume 1. Cambridge University Press.

Wightman, A. S. (1964). La théorie quantique locale et la théorie quantique des champs. In Annales de l'IHP Physique théorique, Volume 1, pp. 403-420.

Yennie, D. R., S. C. Frautschi, and H. Suura (1961). The infrared divergence phenomena and high-energy processes. Annals Phys. 13, 379-452. 